\title{
E2 Transacylase-deficient (Type II) Maple Syrup Urine Disease \\ Aberrant Splicing of E2 mRNA Caused by Internal Intronic Deletions and Association with Thiamine-responsive Phenotype
}

\author{
Jacinta L. Chuang, ${ }^{\star}$ Rody P. Cox, ${ }^{\ddagger}$ and David T. Chuang ${ }^{\star}$ \\ $*$ Department of Biochemistry and ${ }^{\ddagger}$ Department of Internal Medicine, University of Texas Southwestern Medical Center, Dallas, \\ Texas 75235
}

\begin{abstract}
Maple syrup urine disease (MSUD) or branched-chain $\alpha$-ketoaciduria is an autosomally inherited disorder in the catabolism of branched-chain amino acids leucine, isoleucine, and valine. The disease is characterized by severe ketoacidosis, mental retardation, and neurological impairments. MSUD can be classified into genetic subtypes according to the genes of the branched-chain $\alpha$-ketoacid dehydrogenase (BCKD) complex which are affected in patients. We describe here four intronic deletions and an intronic nucleotide substitution in the E2 transacylase gene of type II MSUD, in which the E2 subunit of the BCKD complex is deficient. These new E2 mutations comprise an internal 3.2kb deletion in intron 4 (causing a 17-bp insertion in mRNA), an internal 12-bp (ttaccttgttac) deletion in intron 4 (creating a 10-bp insertion), a 10-bp (catttctaG) deletion in intron 10/ exon 11 junction (leading to a 21-bp deletion), a 2-bp deletion in the exon 5/intron 5 junction (ATgt $\rightarrow \mathrm{A}-\mathrm{t}$ ) (resulting in the skipping of exon 5), and a $G$ to $A$ transition at nucleotide -7 of intron 9 (causing a 6-bp insertion). These intronic mutations were initially detected by secondary alterations in the mutant E2 mRNA, as a result of aberrant splicing. The 3.2-kb deletion in intron 4 was determined by the amplification of the entire intron from both a normal subject $(11.2 \mathrm{~kb})$ and a homozygous patient $(8 \mathrm{~kb})$ by long PCR, followed by subcloning and sequencing of regions flanking the deletion. Similar methods were used to identify and characterize the other intronic alterations. Our results depict heretofore undescribed splicing errors caused by the deletion of internal intronic segments, and provide an approach for detecting this class of novel and rare human mutation. The association of the thiamine-responsive phenotype with a subset of the type II MSUD patients studied is also discussed. (J. Clin. Invest. 1997. 100:736-744.) Key words: branched-chain $\alpha$-ketoacid dehydrogenase complex • secondary insertions/deletions • cryptic splice site activation • new splice site creation • exon skipping
\end{abstract}

Address correspondence to Dr. David T. Chuang, Department of Biochemistry, University of Texas Southwestern Medical Center, 5323 Harry Hines Boulevard, Dallas, TX 75235-9038. Phone: 214-6482457; FAX: 214-648-8856; E-mail: Chuang01@utsw.swmed.edu

Received for publication 4 February 1997 and accepted in revised form 2 May 1997.

J. Clin. Invest.

(C) The American Society for Clinical Investigation, Inc. 0021-9738/97/08/0736/09 \$2.00

Volume 100, Number 3, August 1997, 736-744

http://www.jci.org

\section{Introduction}

Maple syrup urine disease (MSUD) ${ }^{1}$ or branched-chain $\alpha$-ketoaciduria is an autosomal recessively inherited deficiency in the mitochondrial branched-chain $\alpha$-ketoacid dehydrogenase (BCKD) complex. The biochemical basis of this disease is the inability to metabolize branched-chain $\alpha$-ketoacids (BCKA) derived from the essential branched-chained amino acids (BCAA) leucine, isoleucine, and valine. The elevated BCAAs and BCKAs may have severe clinical consequences including ketoacidosis, mental retardation, and neurological impairment. Variations in clinical presentations have led to the classification of MSUD into five clinical phenotypes, i.e., classic, intermediate, intermittent, thiamine-responsive, and E3-deficient forms $(1,2)$. The classic form, which accounts for $75 \%$ of MSUD patients, is manifested within the first $2 \mathrm{wk}$ of life by poor feeding, lethargy, seizures, coma, and death if left untreated. Intermediate MSUD is associated with elevated BCAAs and BCKAs, with progressive mental retardation, and developmental delay without a history of catastrophic illness. An intermittent form of MSUD has normal levels of BCAAs, normal intelligence and development until a stress, e.g., infection, precipitates decompensation with ketoacidosis without seriously affecting intelligence and development. Thiamineresponsive MSUD is similar to the intermediate or intermittent phenotype, but responds to pharmacologic doses of thiamine with normalization of the level of BCAAs (2). The E3-deficient MSUD is caused by defects in the dihydrolipoyl dehydrogenase (E3) component of the BCKD complex that is common to the pyruvate and $\alpha$-ketoglutarate dehydrogenase complexes. Patients with E3 deficiency have dysfunction of all three enzyme complexes and usually die in infancy with severe lactic acidosis (3).

The mammalian BCKD complex is a mitochondrial multienzyme complex, which catalyzes the oxidative decarboxylation of the three BCKA derived from $\operatorname{BCAA}(4,5)$. The enzyme complex is organized around a cubic core comprising 24 identical dihydrolipoyl transacylase (E2) subunits, to which branched-chain $\alpha$-keto acid decarboxylase (E1), E3, a specific kinase, and a specific phosphatase are attached through ionic interactions. The kinase and the phosphatase are responsible for the regulation of BCKD complex by a reversible phosphorylation (inactivation)/dephosphorylation (activation) mecha-

1. Abbreviations used in this paper: BCAA, branched-chain amino acid; BCKA, branched-chain $\alpha$-ketoacid; BCKD, branched-chain $\alpha$-ketoacid dehydrogenase; E1, branched-chain $\alpha$-ketoacid decarboxylase; E2, dihydrolipoyl transacylase; E3, dihydrolipoyl dehydrogenase; IVS, intervening sequences; MSUD, maple syrup urine disease; RT, reverse transcription. 
nism (6). The E1 component is a heterotetramer consisting of two E1 $\alpha$ and two E1 $\beta$ subunits. The E3 component, a flavoprotein, is a homodimer. Therefore, there are in total six genetic loci that contribute to the BCKD complex, and mutations in all four different catalytic subunits (E1 $\alpha, E 1 \beta, \mathrm{E} 2$, and E3) have been described in MSUD patients. Genetic subtypes of MSUD have been proposed on the basis of the subunit affected (1). These include type IA MSUD affecting the E1 $\alpha$ subunit; type IB affecting the E1 $\beta$ subunit; type II, affecting the E2 subunit; and type III, affecting the E3 subunit. Types IV and V are reserved for MSUD involving the kinase and the phosphatase, respectively, and have not been reported.

The E2 gene perturbed in type II MSUD is located on chromosome 1p31 (7). The gene contains 11 exons and spans $68 \mathrm{~kb}(8)$. The E2 gene product comprises three independently folded domains, i.e., lipoyl-bearing, E1/E3-binding, and innercore domains which are connected by flexible hinge regions. This domain structure is highly conserved between different E2 proteins of the $\alpha$-ketoacid dehydrogenase complexes. Several mutations in the E2 gene of type II MSUD patients have been described, including a 17-bp insertion $5^{\prime}$ to exon 5 , a $\mathrm{T}$ to $\mathrm{G}$ transversion corresponding to an F215C substitution in exon 7 from a thiamine-responsive patient, WG-34 (9), and a 2-bp deletion in exon 2 and a $\mathrm{G}$ to $\mathrm{T}$ transversion (E163X) in exon 6 (10). A G to A transition at the last nucleotide of exon 8 (11) and a 1-bp deletion at the $5^{\prime}$ donor site of intron 8 (12) were also described, resulting in the skipping of exon 8 .

To further elucidate relationships between the genotype and biochemical mechanisms underlying MSUD, we have un- dertaken an investigation to identify additional mutations in type II MSUD. In this communication, we report five new mutations in the E2 gene which cause type II MSUD. It is noteworthy that a significant number of these mutations involve rare deletions of internal intronic segments which lead to secondary insertions/deletions in the transcript through utilization of cryptic or new splice sites. The occurrence of these insertion/deletion mutations was initially found by reverse transcription (RT)-PCR and direct sequencing of the mutant E2 mRNA. A 3.2-kb internal deletion in intron 4 was established by amplification and analysis of the entire intron (normal size: $11.2 \mathrm{~kb}$ ). Moreover, there is an apparent correlation of type II MSUD mutations with the thiamine-responsive phenotype. The significance of this correlation in the treatment of type II patients is discussed.

\section{Methods}

Cell cultures and cell lines. Lymphoblast and fibroblast cell cultures were grown as described previously (13). Fibroblasts or lymphoblasts prepared from blood specimens by infection with EBV (14) were used for analysis. The following is a list of physicians who provided patients' blood samples or fibroblasts for studies: C.E. (fibroblasts), Dr. Juan Sotos (Children's Hospital, Columbus, OH); W.J. (lymphoblasts), Dr. Selma Snyderman (Mount Sinai School of Medicine, New York, NY); S.B. (fibroblasts), Dr. Susan Berry (Children's Hospital, Minneapolis, MN); A.E. (lymphoblasts), Dr. Grant Mitchell (Hôpital Sainte-Justine, Montreal, Quebec); S.G. (fibroblasts and lymphoblasts), Dr. Louis Dallaire (Hôpital Sainte-Justine, Montreal, Que-

Table I. Oligonucleotide Primers for Amplification of Human E2 Gene and RT-PCR of E2 mRNA

\begin{tabular}{|c|c|c|c|c|}
\hline Primer name & Exon/cDNA amplified & Primer sequence & Direction & Base number/intronic sequence \\
\hline $1 \mathrm{~F}$ & 1 & $5^{\prime}$ - TCTTTTCACTCCCCGCT-3' & + & $(-) 184-(-) 168$ \\
\hline $1 \mathrm{R}$ & & $5^{\prime}-$ TCAGATCTGCCCAAAC - 3' & - & Intronic \\
\hline $2 \mathrm{~F}$ & 2 & $5^{\prime}$ - GAGATAAGCCGGTATGGTTG - 3' & + & Intronic \\
\hline $2 \mathrm{R}$ & & 5' - CCGGCTAGAAATACACAGTC - 3' & - & Intronic \\
\hline $3 \mathrm{~F}$ & 3 & $5^{\prime}-$ TGCCTCTGCCTGAGAACATT - 3' & + & Intronic \\
\hline $3 \mathrm{R}$ & & 5' - AATTTAAATGGATTCCCACTATCC - 3' & - & Intronic \\
\hline $4 \mathrm{~F}$ & 4 & 5' - CATCCTTAATATGATCTGTACTTATGACTG - 3' & + & Intronic \\
\hline $4 \mathrm{R}$ & & 5' - TCACTTTTAATTGGGACCCAATGAC - 3' & - & Intronic \\
\hline $5 \mathrm{~F}$ & 5 & 5' - сCСTCTCCCATACCATTAGGATAGTGTG- 3' & + & Intronic \\
\hline $5 \mathrm{R}$ & & 5' - GTACACTTCCTATACAATCTCAGAC - 3' & - & Intronic \\
\hline $6 \mathrm{~F}$ & 6 & 5' - ACTGTCAGTTGATCAAATTCC - 3' & + & Intronic \\
\hline $6 \mathrm{R}$ & & 5' - CTACTACTGAGGTAGCTTCC - 3' & - & Intronic \\
\hline $7 \mathrm{~F}$ & 7 & 5' - AAAGGGTTTAACTGAGAG - 3' & + & Intronic \\
\hline $7 \mathrm{R}$ & & 5' - AAGAGCAAAACTCTGTCTC - 3' & - & Intronic \\
\hline $8 \mathrm{~F}$ & 8 & 5' - GTTTCAAATTCTCTATCCTTTCTAAG - 3' & + & Intronic \\
\hline $8 \mathrm{R}$ & & 5' - CAAGCACATTTGCCCCATCTTCT - 3' & - & Intronic \\
\hline $9 \mathrm{~F}$ & 9 & 5' - CTAGATGGCAGTGAAGGTGATCCT - 3' & + & Intronic \\
\hline 9R & & $5^{\prime}-$ CCAGAGGAACAAATGCCAAGTGAC - 3' & - & Intronic \\
\hline $10 \mathrm{~F}$ & 10 & 5' - AGTCACTTGGCATTTGTTCCC - 3' & + & Intronic \\
\hline $10 \mathrm{R}$ & & 5' - CTCTTCATTGTGTTAGTCCCTG- 3' & - & Intronic \\
\hline $11 \mathrm{~F}$ & 11 & 5' - GCTTGAGCTCTGAACAAGTG - 3' & + & Intronic \\
\hline hE2-3' & & 5' - GCTCAAAAAGTTCAAGAATGTCTTATCAGT - 3' & - & $1638-1667$ \\
\hline $5^{\prime}$ primer & cDNA & $5^{\prime}-$ GCTGCAGTCCGTATGCTGA - 3' & + & $18-36$ \\
\hline $3^{\prime}$ primer & & 5' - CTAGTAGCATAAAAGCTGGG - 3' & - & $1453-1434$ \\
\hline
\end{tabular}

The exon number is assigned according to the structure of the human E2 gene (8). Nucleotide positions in the coding region are based on the complete human E2 cDNA sequence (27). + refers to the $5^{\prime} \rightarrow 3^{\prime}$ orientation and - the $3^{\prime} \rightarrow 5^{\prime}$ orientation. 
bec); and MGF-497 (fibroblasts), Dr. Vivian Shih (Massachusetts General Hospital, Boston, MA).

Reverse transcription and amplification of E2 $m R N A$ and sequencing. For RT-PCR, the first strand cDNA was reverse-transcribed from $10 \mu \mathrm{g}$ of total RNA from fibroblasts or lymphoblasts derived from patients or normal subjects using primer hE2-3' (Table I) and the AMV reverse transcriptase (Promega, Madison, WI). The entire E2 cDNA was amplified using the $5^{\prime}$ and $3^{\prime}$ primers (Table I) flanking the coding region. Direct sequencing of amplified cDNA was carried out using the Promega $\mathrm{fmol}^{\mathrm{TM}}$ DNA sequencing system according to the supplier's instructions. For compound-heterozygous mutations, the amplification product was subcloned and doublestranded sequencing was performed (15).

Genomic amplification of E2 exons and sequencing. For genomic amplification, the entire coding region of each exon and intron/exon boundary was amplified from genomic DNA prepared from normal or patient's cells using Taq DNA polymerase from Promega. The sense and antisense primers used for amplification of the $11 \mathrm{E} 2$ exons are shown in Table I. Direct sequencing of amplified products, subcloning, and double-stranded sequencing were as described above.

Amplification of intron 4. The entire intron 4 (11.2 kb in length) of the human E2 gene was amplified from genomic DNA of lymphoblasts using the Expand ${ }^{\mathrm{TM}}$ long template PCR system from Boehringer-Mannheim (Mannheim, Germany). The sense and antisense primers are located in exon 4 and exon 5, respectively, with the following sequences: 5'-GCTTCTGTTACCATCACTAGTCGTTATGATGG-3' (sense) (bases 330-365) and 5'-CTCTTGGTGTGTATGTTCATCATGAGACACTGCAG-3' (antisense) (bases 509-475). The amplification mixture contained $50 \mathrm{mM}$ Tris- $\mathrm{HCl}, \mathrm{pH} 9.2,350$ $\mu \mathrm{m}$ of the dNTP mixture, $300 \mathrm{nM}$ each of the sense and antisense primers, $16 \mathrm{mM}\left(\mathrm{NH}_{4}\right)_{2} \mathrm{SO}_{4}, 1.75 \mathrm{mM} \mathrm{MgCl}_{2}, 0.75 \mu \mathrm{l}$ of the Expand ${ }^{\mathrm{TM}}$ enzyme mixture, and $500 \mathrm{ng}-1 \mu \mathrm{g}$ of genomic DNA in a final volume of $50 \mu \mathrm{l}$. Amplification was carried out in a thin-walled reaction tube on a programmable thermal controller (model PTC-100; MJ Research Inc., Watertown, MA) with denaturation of the template at $93^{\circ} \mathrm{C}$ for $2 \mathrm{~min}$. This was followed by 10 cycles of denaturation at $93^{\circ} \mathrm{C}$ for $30 \mathrm{~s}$, and annealing and elongation at $68^{\circ} \mathrm{C}$ for $10 \mathrm{~min} .20 \mathrm{more} \mathrm{cy-}$ cles were performed with denaturation at $93^{\circ} \mathrm{C}$ for $30 \mathrm{~s}$, annealing at $68^{\circ} \mathrm{C}$ for $30 \mathrm{~s}$, and elongation at $68^{\circ} \mathrm{C}$ for $10 \mathrm{~min}$, with a 20 -s increment for elongation in each cycle.

Other procedures. Western blotting was performed as described previously (16). Activity of the BCKD complex in fibroblasts or lymphoblasts was determined by measuring $\mathrm{CO}_{2}$ release when intact cells were incubated in $\alpha$-keto[ $\left[1-{ }^{14} \mathrm{C}\right]$ isovalerate $(10,13)$. The heteroduplex analysis for mutations was carried out as described previously (17).

\section{Results}

Identification of the type II MSUD cells. Lymphoblasts or fibroblasts derived from suspected MSUD patients were assayed for the rate of decarboxylation of $\alpha$-keto[ $\left.1-{ }^{14} \mathrm{C}\right]$ isovalerate by the intact cell assay. MSUD was confirmed by either markedly reduced or no decarboxylation of the $\alpha$-ketoacid when the results with patients' cells were compared to those with normal. To localize the subunit of the BCKD complex affected, cell lysates from established MSUD patients were subjected to Western blotting using antibody to E1 $\alpha, \mathrm{E} 1 \beta$, or E2 subunits as a probe. The type II MSUD cells used in this study showed either the absence or markedly lower than normal levels of E2 protein (data not shown).

Exonic insertions/deletions caused by internal deletions of intronic segments. We recently studied a type II MSUD patient (S.G.), in which we were unable to amplify exon 5 of the E2 gene using a pair of primers (5F and $5 \mathrm{R}$, Table I) flanking exon 5. RT-PCR of the total RNA prepared from lympho-
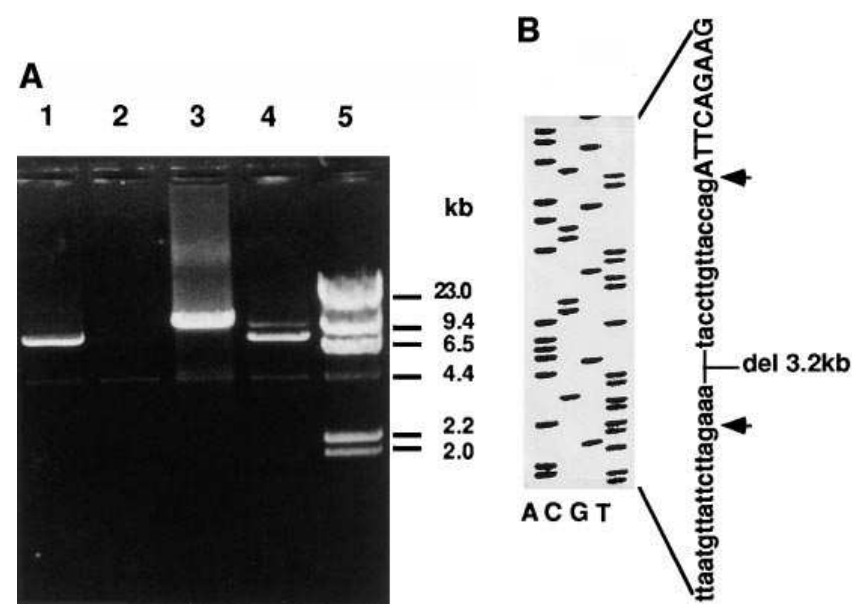

\section{C}

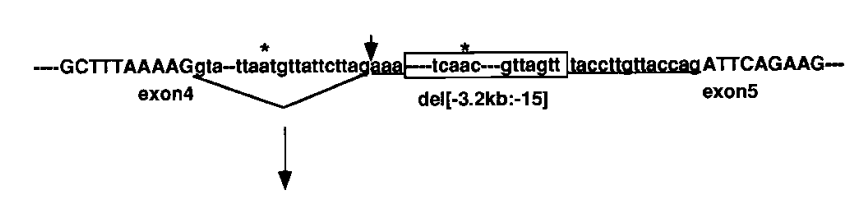

mRNA

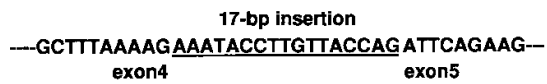

Figure 1. Amplification of intron 4 showing a 3.2-kb intronic deletion in the IVS4del[-3.2 kb:-15] allele which produces aberrant splicing. (A) Size determination of PCR product. Ethidium bromide stains of a gel showing that normal intron 4 (lane 3 ) is $11.2 \mathrm{~kb}$. Intron 4 from a homozygous type II MSUD patient S.G. (lane 1) is $8.0 \mathrm{~kb}$ in size. The exact lengths of amplified introns were estimated by adding sizes of EcoRI-digested fragments (data not shown). Lane 2, No DNA template added. Lane 3, Amplified intron 4 from a normal subject. Lane 4 , Amplified intron 4 from a heterozygous type II MSUD patient, S.B., who has both a normal intron 4 sequence and 3.2-kb deletion from intron 4. Lane 5, Size markers of HindIII-digested $\lambda$ DNA fragments. The weak 4.4-kb bands that appear in all lanes are nonspecific PCR product. (B) Sequencing gel showing the $3.2-\mathrm{kb}$ deletion in intron 4 . The normal $(11.2 \mathrm{~kb})$ and the mutant $(8.0 \mathrm{~kb})$ were amplified by long PCR, and the amplified DNA digested with EcoRI. Southern blot analysis showed that a 300-bp EcoRI fragment from the mutant PCR product hybridized strongly with an oligonucleotide probe corresponding to the 17-bp insert in the mutant E2 mRNA. This DNA fragment was isolated, subcloned, and sequenced. Nucleotide sequences flanking the $3.2-\mathrm{kb}$ deleted region $($ del $3.2 \mathrm{~kb})$ are shown. The upper and lower arrows depict the original and new 3 '-splice sites, respectively. Normal sequences in this region are shown in Fig. $2 \mathrm{~A}$ (lanes 2 and 3). The two MSUD patients (N.R. and L.J.) are not affected in this region of the E2 gene. $(C)$ Proposed mechanism for the 17-bp insertion in the mutant E2 mRNA. The 3.2-kb deletion occurs immediately $5^{\prime}$ to the 14-bp intronic sequence present in the E2 transcript. The aaa trinucleotides that are also present in the 17-bp insert are located between the $3.2-\mathrm{kb}$ deletion and the invariant ag dinucleotides that are used as a cryptic $3^{\prime}$ acceptor splice site. The lowercase letters represent intronic sequences. The uppercase letters refer to exonic or mRNA sequences. The 17-bp inserted intronic sequence in the mRNA is underlined. The original (base -29) and the new branch point based on a consensus sequence YTRAY (25) are indicated by asterisks. 
blasts and direct sequencing revealed that S.G. is homozygous for the 17-bp insertion (5' AAATACCTTGTTACCAG 3') which was shown previously to occur in compound-heterozygote thiamine-responsive patient WG-34 $(2,9)$. Intron 4 of the E2 gene was subsequently amplified using genomic DNA from normal and from S.G. cells as templates and using a sense and an antisense primer located in exon 4 and exon 5, respectively (see Methods). The approximate sizes of PCR products were estimated by adding the sizes of EcoRI-digested fragments of the amplified DNA (data not shown). An 11.2-kb PCR product corresponding to intron 4 was obtained with genomic DNA from normal cells as template (Fig. $1 A$, lane 3 ). In contrast, an 8 -kb fragment corresponding to intron 4 was generated with genomic DNA from the homozygous patient S.G. (lane 1), which indicated a $3.2-\mathrm{kb}$ deletion in the intron. This allele from S.G. is designated IVS4del [-3.2 kb:-15]. Amplification of the intron 4 from another type II MSUD patient (S.B.) showed both the normal 11.2-kb fragment and the truncated $8-\mathrm{kb}$ fragment, indicating that the patient is compoundheterozygous for the IVS4del[-3.2 kb:-15] allele (lane 4). The lower intensity of the $11.2-\mathrm{kb}$ band in S.B. is probably due to a preferential amplification of the shorter $8-\mathrm{kb}$ fragment during long PCR. The intronic sequences flanking the deleted 3.2-kb region have been determined (Fig. $1 B$ ). Based on these sequences, a mechanism for the 17-bp insertion in mutant E2 mRNA is shown in Fig. 1 C. According to this mechanism, a new $3^{\prime}$ acceptor site (arrow) upstream of the intact original site is used, which causes insertion (underlined) of the 14-bp intronic sequence and the determined $a a a$ intronic triplet in the mutant E2 mRNA from S.G. (Fig. $1 C$ ). Since no normal E2 mRNA was detected by direct sequencing of amplified cDNAs, the original $3^{\prime}$-splice site is not used in the aberrant splicing.

A

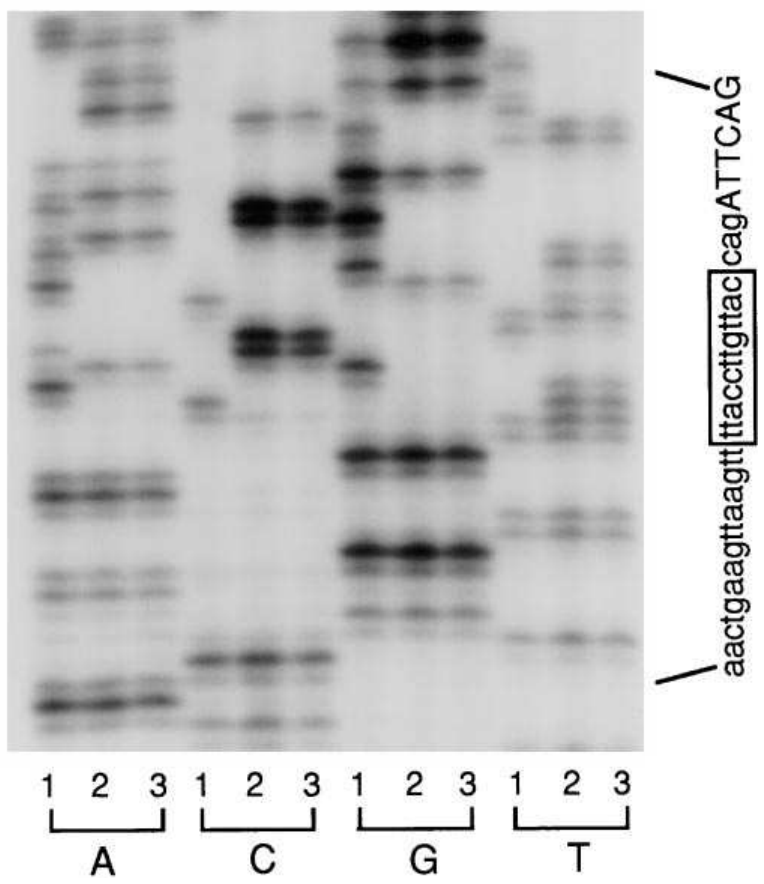

Type II MSUD mutations in the compound-heterozygous patient S.B. were initially studied by amplifying the 11 exons of the E2 gene from this subject. Heteroduplex analysis of amplified E2 exons indicated possible mutations in exon 5. Amplification and direct sequencing of the exon 5 region showed an apparent homozygous 12-bp (ttaccttgttac) deletion in the 3 '-terminal region of intron 4 (Fig. $2 A$ ). However, RT-PCR of the E2 mRNA followed by direct sequencing showed a heterogeneous sequence pattern after exon 4. Subcloning of the RT-PCR products and subsequent sequencing showed the presence of only two types of mutant E2 mRNA. One contained the above 17-bp insertion $5^{\prime}$ to exon 5, indicating the presence of the IVS4del[-3.2 kb:-15] allele. The second mutant transcript harbored a novel 10-bp insertion (TTAAGTTCAGA), also 5' to exon 5. The results explained the apparent homozygosity of S.B. observed in exon amplification. The exon 5 region of the IVS4del[ $-3.2 \mathrm{~kb}:-15]$ allele in S.B. could not be amplified, since the sense primer used was located in the deleted 3.2-kb intronic segment. The 12-bp intronic deletion (Fig. 2 B, boxed) causes the sole utilization of a new $3^{\prime}$-splice site (arrow), resulting in the insertion of $10 \mathrm{bp}$ of the downstream $3^{\prime}$-intronic sequence (underlined). This allele is named IVS4del[-15:-4].

Based on the absence of the E2 subunit on Western blotting, it was determined that patient W.J. had type II MSUD. RT-PCR was carried out on total RNA of lymphoblasts prepared from this patient. Direct sequencing of the PCR product showed a homozygous 21-bp deletion (GCCATTCCCCGATTTAACCAG), proximal to the 5'-terminus of exon 11. No normal E2 mRNA was present. Genomic DNA from W.J. was amplified using a sense primer located upstream (11F, Table I) of the intron 10/exon 11 splice junction and an antisense primer in the noncoding region of exon 11 (hE2-3').

B

GENE

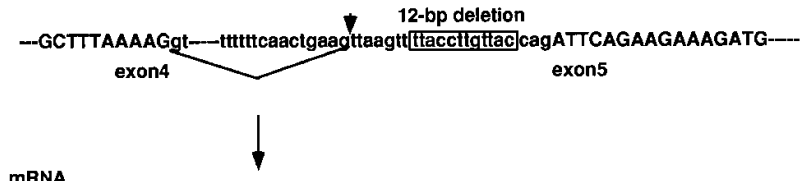

mRNA

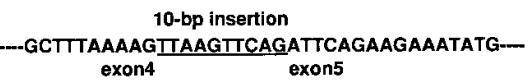

Figure 2. A 12-bp deletion in intron 4 of the IVS4del[-15:-4] allele resulting in a 10-bp insertion in mutant E2 mRNA. (A) Sequencing gel showing the 12-bp deletion. Exon 5 region was amplified from genomic DNA of three type II MSUD patients using primers 5F and 5R (Table I). Sequencing samples were applied in groups according to the nucleotides A, C, G, and T. Lane 1, patient S.B.; lane 2, patient N.R.; lane 3, patient L.J. Boxed sequences are the deleted sequence of $12 \mathrm{bp}$ from intron 4. S.B. is a heterozygote for the 12-bp intronic deletion. The other allele contains the 3.2-kb deletion in intron 4 (Fig. 1), and was not amplified because the sense primer was in the deleted region. The other two MSUD patients are normal in this region. $(B)$ Utilization of a new $3^{\prime}$ acceptor splice site (arrow) upstream of the normal in intron 4 results in a 10-bp insertion (underlined) in the mutant E2 mRNA. The lowercase letters refer to the intronic sequences, and uppercase letters are exonic or mRNA sequences. 


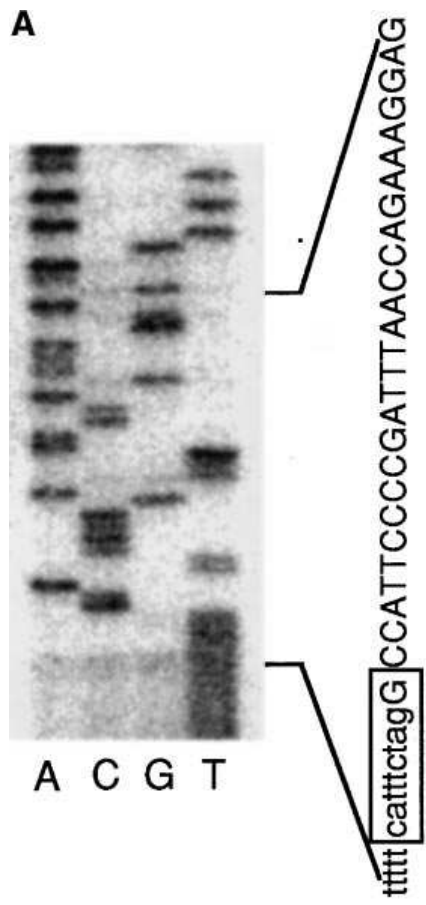

B

GENE

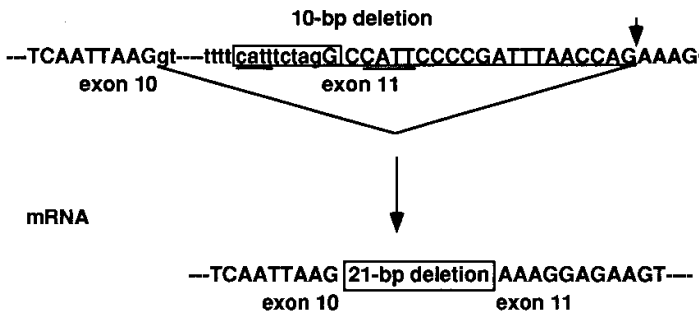

Figure 3. A 10-bp deletion in intron 10 in the IVS10del[-9:+1] allele which produces a 21 -bp deletion in the mutant $\mathrm{E} 2 \mathrm{mRNA}$. (A) Sequencing gel showing the 10-bp deletion (boxed sequence cattctag $G$ ). The region containing the exon 11 region was amplified from genomic DNA of a type II MSUD patient, W.J., and the PCR product subjected to direct sequencing. (B) Activation of a cryptic $3^{\prime}$ acceptor splice site (arrow) which results in a 21-bp deletion (underlined) in the mutant E2 mRNA. The two tandem direct repeats (catt and CATT) which cause slipped mispairing and subsequent gene deletion (21) are underlined.
Direct sequencing of the amplified genomic DNA indicated that the $9 \mathrm{bp}$ at the $3^{\prime}$-end of intron 10 including the invariant $3^{\prime}$ ag splice site and one terminal $\mathrm{G}$ nucleotide of exon 11 (catttctagG) were deleted (Fig. $3 A$ ). This deletion completely abolishes the normal $3^{\prime}$-splice site of intron 10 , which leads to the activation of a cryptic $3^{\prime}$-splice site (the original site is destroyed) located in exon 11 (Fig. $3 \mathrm{~B}$, arrow). This aberrant splicing results in the elimination of $21 \mathrm{bp}$ (Fig. 3 B, boxed) from the $5^{\prime}$-end of exon 11 in the E2 mRNA of this patient. The allele is designated IVS10del[-9:+1].

A thiamine-responsive type II MSUD patient, C.E., was previously shown to be a compound-heterozygote for a 2-bp deletion allele (90delAT) in exon 2 of the E2 locus (10). RT-PCR of the E2 mRNA was carried out with lymphoblastoid cells from this patient. Sequencing of subcloned PCR product disclosed that exon 5 was frequently skipped (10). A pair of primers flanking exon 5 were therefore used to amplify this region with genomic DNA from C.E. as template. Sequencing of the subcloned PCR products revealed a deletion of $2 \mathrm{bp}$ at the exon 5/intron 5 junction (ATgt $\rightarrow \mathrm{A}-\mathrm{t}$ ) of one allele (Fig. $4 A$ ). The allele is designated IVS5del[-1:+1]. The deletion of the invariant $5^{\prime}$-splice site results in the skipping of exon 5 (Fig. $4 \mathrm{~B}$ ).

A classic MSUD patient, A.E., was the progeny of consanguineous Moroccan parents. He and an older brother both had episodes of lactic acidosis and died of progressive neurological symptoms. RT-PCR of A.E.'s E2 mRNA and direct sequencing revealed that the patient was homozygous for a 6-bp insertion derived from the $3^{\prime}$-terminal sequence of intron 9. The normal E2 mRNA was not detected. Subsequently, the sequence of intron 9 upstream of the $3^{\prime}$-splice site was amplified and directly sequenced. The results showed an A to $\mathrm{G}$ transition at nucleotide -7 of intron 9 (Fig. $5 A$ ). This causes the creation of a new $3^{\prime}$-splice site, resulting in an invariant retention of the 6-bp intronic sequence (Fig. $5 \mathrm{~B}$ ). The inserted bases harbor a stop codon TAG immediately $5^{\prime}$ to exon 10 , which causes the absence of the E2 subunit.

\section{Discussion}

This report describes five intronic mutations in type II MSUD which produce secondary insertion/deletion or exon skipping in the mutant E2 transcripts. Some of these alterations in the E2 mRNA are the result of splicing errors by a novel mechanism. The majority of the splicing errors reported to date are associated with mutations in the $5^{\prime}$ and $3^{\prime}$-splice sites. Of the $5^{\prime}$-splice site mutations, $60 \%$ involve the invariant $g t$ dinucleotide. Among the $3^{\prime}$-splice site mutations, $87 \%$ perturb the invariant ag nucleotide pair $(18,19)$. However, aberrant splicing caused by deletion of internal intronic segments, as shown in this study, has not been described. The absence of this mechanism in the literature is probably due to inherent difficulties in detecting internal intronic deletions especially with large introns (see below). It is noteworthy that 5 (this study) out of the 19 currently known E2 mutations involve deletions or base substitutions in the introns (20). The basis for this relatively high frequency of intronic alteration in type II MSUD is mostly unknown. In the case of the IVS10del[-9:+1] allele, however, the 10-bp deletion is associated with a pair of tandem direct repeats, which occur either within (intronic catt) or $3^{\prime}$ to (exonic CATT) the deleted bases (Fig. $3 \mathrm{~B}$, underlined). The data strongly suggest slipped mispairing during DNA replication as a mechanism for the gene deletion, as proposed by Krawczak and Cooper (21). In contrast, the 15 known mutations in the E1 $\alpha$ gene in type IA MSUD occur only in the exons (20).

The intronic mutations described in this study were first suggested by amplification of the mutant E2 mRNA, followed by sequencing of cDNA subclones (9) or direct sequencing of the RT-PCR product. For example, the IVS4del[-3.2 kb:-15] allele produces a 17-bp insertion in the E2 mRNA immediately $5^{\prime}$ to exon 5 . The insertion was initially detected in a cDNA subclone from type II MSUD patient WG-34, who was a compound heterozygote for this allele (9). However, the au- 
A

normal

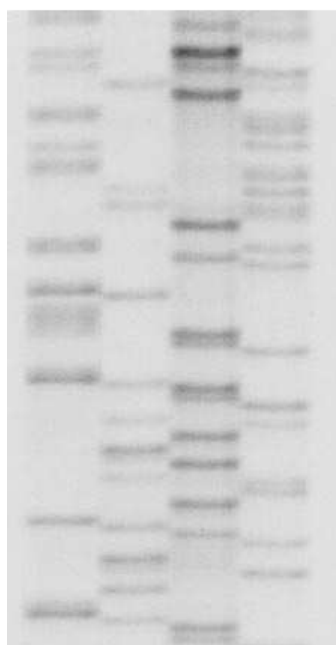

A $\quad C \quad G \quad T$ mutant

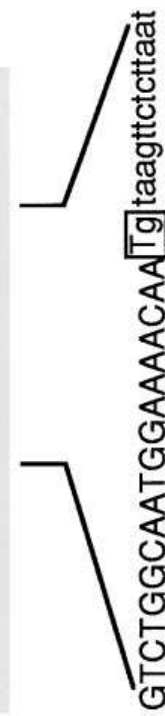

B

GENE

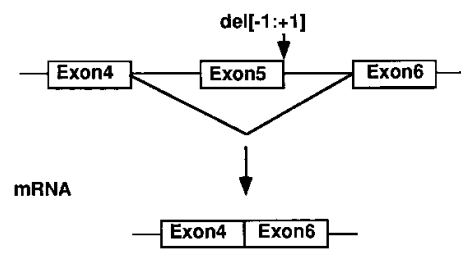

Figure 4. A 2-bp deletion in the IVS5del[ -1 : +1 ] allele resulting in the skipping of exon 5. $(A)$ Sequencing gel showing the 2-bp (Tg) deletion (boxed) at base -1 and +1 of the exon $5 /$ intron 5 junction. The exon 5 region of the E2 gene encompassing the splice junction was amplified from genomic DNA of a normal subject and a type II MSUD patient, E.C. The PCR products were subcloned and subjected to double-stranded sequencing. (B) Aberrant splicing resulting in the skipping of exon 5 . The 2-bp deletion at the exon 5 /intron 5 junction is indicated by del[ $[-1:+1]$. thenticity of the 17-bp insertion allele was questioned because of the failure to show intronic alterations, when intron 4 of WG-34 was amplified with the primer pair 5F and 5R (Table I). We were later unable to amplify exon 5 from a type II MSUD patient, S.G., who was homozygous for the 17-bp insertion at the mRNA level. The question then became whether this patient had an intronic deletion which encompassed the sense primer $(5 \mathrm{~F})$ region. This prompted the amplification of the entire intron 4 from a normal subject $(11.2 \mathrm{~kb})$ and S.G. by long PCR. Subcloning and sequencing of restriction fragments of the PCR products identified a 3.2-kb internal segment of intron 4 which was truncated in the homozygous IVS4del[ -3.2 kb:-15] allele of S.G. Thus, our studies demonstrate the use of long PCR in analyzing internal intronic mutations and emphasize the importance of RT-PCR of mutant mRNA as a first step in detecting secondary insertions/deletions caused by internal intronic alterations. However, there are limitations in the approach of RT-PCR of mRNA. For example, certain exonic alterations generate nonsense mutations in early coding regions, rendering the mRNA unstable (22). In this case, amplification and direct sequencing of exons from genomic DNA is necessary to detect the exonic mutations (22).

A common feature of the intronic mutations described in this study involves aberrant splicing through the activation of cryptic splice sites (when the original site has been destroyed) or the creation or utilization of new splice sites (when the original site remains intact) in the E2 gene. The 3.2-kb deletion in intron 4 of the IVS4del[ $-3.2 \mathrm{~kb}:-15]$ allele results in the use of a new $3^{\prime}$-splice site upstream of the original $3^{\prime}$-splice site and of the truncated region. The aberrant splicing supports
A

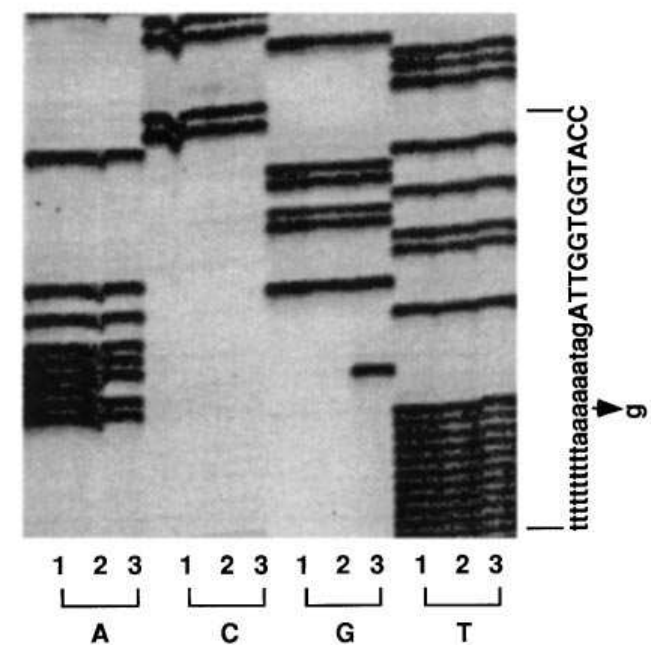

B

GENE

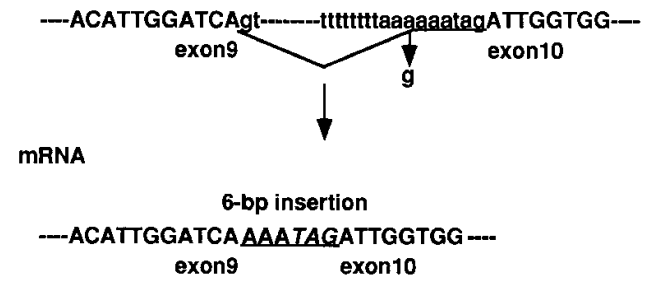

Figure 5. An a to g transition in intron 9 of the IVS9-7A $\rightarrow$ G allele causing a 6-bp insertion in the mutant mRNA. $(A) \mathrm{Se}$ quencing gel showing the a to $g$ transition. Intron 9 from three type II MSUD patients, Z.G. (lane 1), RD1 (lane 2), and A.E. (lane 3), was amplified and subjected to direct sequencing. DNA samples were applied in groups according to nucleotide A, C, G, and T. The homozygous a to $\mathrm{g}$ intronic transition in A.E. (lane 3) is indicated by an arrow. Z.G. and RD1 are normal in this region. $(B)$ The

mechanism for the 6-bp insertion. The a to $g$ transition produces a new $3^{\prime}$-splice site which is used in aberrant splicing. This results in the retention of 6-bp intronic sequence including a TAG inframe stop codon in the mRNA. 
Table II. Consensus Scores of 3'-Splice Sites in Normal and Mutant E2 Alleles

\begin{tabular}{lclc}
\hline \multicolumn{1}{c}{ Allele } & Intron No. & 3'-Splice site* & Consensus score \\
\hline \multirow{2}{*}{ Normal } & & & $\%$ \\
IVS4del [-3.2 kb:-15] & 4 & Original & 84.6 \\
& 4 & Original & 84.6 \\
IVS4del [-15:-4] & 4 & New & 73.5 \\
& 4 & Original & 66.4 \\
Normal & 4 & New & 79.5 \\
IVS9-7A $\rightarrow$ G & 9 & Original & 70.8 \\
& 9 & Original & 70.8 \\
Normal & 9 & New & 85.0 \\
IVS10del [-9:+1] & 10 & Original & 91.5 \\
& 10 & Deleted & - \\
& 10 & Cryptic & 75.5 \\
\hline
\end{tabular}

*The new 3 '-splice site refers to the alternate site which is used when the original $3^{\prime}$-splice site is still intact. The cryptic $3^{\prime}$-splice site denotes the alternate site which is activated after the original site is destroyed as a result of the mutation (19). ${ }^{*}$ The consensus score was calculated by assigning a score to each of the 14 nucleotides flanking the invariant $a g$ dinucleotide in the allele, according to compatibility of the base to the consensus sequence (25). A $100 \%$ consensus score indicates complete compatibility and the maximal strength of the splice site.

and extends the theory of exon definition as a guide for the splice site selection. The concept of exon definition dictates that in the pre-mRNA with large introns, the splicing machinery searches for a pair of closely spaced splice sites in an exonic polarity (23). This mechanism imposes a size constraint for internal exons, which can be no larger than 400 nucleotides. The new $3^{\prime}$-splice site was not functional in the normal E2 gene because it was far upstream of the $3^{\prime}$-end of exon 5 . The new site was activated only after it was moved closer to exon 5 by the internal deletion of intron 4 in the IVS4del[-3.2 kb:-15] al- lele. The strength of this new $3^{\prime}$-splice site is similar to the original site, as indicated by the consensus scores (24) of 73.5 and $84.6 \%$, respectively (Table II). The fact that the new 3 -splice site is solely used in the mutant allele provides evidence for $5^{\prime} \rightarrow 3^{\prime}$ polarity in exon definition. That is, between the two proximal splice sites of similar strength, the upstream splice site is first encountered by the spliceosome in exon scanning, and is exclusively used for RNA splicing. It should also be mentioned that a putative normal branch point (nucleotide -29) based on a consensus sequence YTRAY (25) is deleted in the IVS4del[-3.2 kb:-15] allele (Fig. $1 C$ ). An alternate branch point $13 \mathrm{bp}$ upstream of the new $3^{\prime}$-splice site renders this site suitable for $3^{\prime}$ splicing.

As for the IVS4del[-15:-4] allele, the 12-bp intronic deletion near the original 3 -splice site in intron 4 reduces the strength of the original site with the consensus score decreasing from 84.6 to $66.4 \%$ (Table II). This results in the sole usage of a new upstream $3^{\prime}$-splice site which has a relatively strong consensus score of $79.5 \%$ (Table II). It is noted, however, that the new and original $3^{\prime}$-splice sites are of similar strength and in close vicinity to each other (20 bp apart). Despite being upstream of the original site, the new site is not used in normal splicing. The reason is not clear, but may suggest additional intronic sequence specificities in influencing the $3^{\prime}$-splice site selection.

In the IVS9-7A $\rightarrow \mathrm{G}$ allele, an $\mathrm{A}$ to $\mathrm{G}$ transition created a new $3^{\prime}$-splice site, which is used entirely over the downstream authentic $3^{\prime}$-splice site. This causes a retention of $6 \mathrm{bp}$ of intron 9 sequence juxtaposed to the $5^{\prime}$-terminus of exon 10 . The consensus score shows $85 \%$ for the new $3^{\prime}$-splice site and $70.8 \%$ for the original site (24). The data again support the concept of exon scanning in $5^{\prime} \rightarrow 3^{\prime}$ polarity, and the occurrence of a stronger $3^{\prime}$-splice site through the A to $\mathrm{G}$ mutation results in an exclusive recognition of the newly created site by the spliceosome. An A to $G$ transition was also reported in intron 16 of the muscle phosphofructokinase gene in a patient with glycogenosis type VII (Tarui disease) (26). This intronic mutation leads to the retention of 63 nucleotides of intron 16 by a mechanism similar to that which occurs in the IVS9-7 $\mathrm{A} \rightarrow \mathrm{G}$ allele of this study. As for the IVS10del[-9:+1] allele, a

Table III. Summary of Genotypes, Enzyme Activity, and Background of MSUD Patients

\begin{tabular}{|c|c|c|c|c|c|}
\hline Patient & Allele* & $\begin{array}{l}\text { BCKD } \\
\text { activity }\end{array}$ & $\begin{array}{c}\text { Residual } \\
\text { E2 protein }\end{array}$ & Clinical phenotype & Background \\
\hline & & $\%$ & & & \\
\hline WG-34 & IVS4del[-3.2 kb:-15]/F215C ‡ & $30-40$ & Yes & $\begin{array}{l}\text { Intermittent/ } \\
\text { thiamine-responsive }\end{array}$ & Unknown \\
\hline S.G. & IVS4del[-3.2 kb:-15] (homozygous) & 0 & No & Classic & French Canadian \\
\hline S.B. & IVS4del[-3.2 kb:-15]/IVS4del[-15:-4] & 0 & No & Classic & Caucasian \\
\hline MGF- $497^{\S}$ & IVS4del[-3.2 kb:-15]/R230G" & $7-14$ & Yes & $\begin{array}{l}\text { Intermittent/ } \\
\text { thiamine-responsive }\end{array}$ & Caucasian \\
\hline W.J. & IVS10del[-9:+1] (homozygous) & $\mathrm{ND}^{\mathbb{1}}$ & No & Classic & American Indian/Spanish \\
\hline C.E. & 90delAT/IVS5del[-1:+1] & 0 & No & $\begin{array}{l}\text { Classic/ } \\
\text { thiamine-responsive }\end{array}$ & German/Dutch/Irish \\
\hline A.E. & IVS9-7A $\rightarrow \mathrm{G}$ (homozygous) & 1 & No & Classic & Moroccan \\
\hline
\end{tabular}

* The allele name conforms to the recommendations of the Ad Hoc Committee on Mutation Nomenclature for the journal Human Mutation (29). ${ }^{\ddagger}$ The F215C missense mutation in exon 7 was previously reported (9). ${ }^{\S} \mathrm{MGF}-497$ was previously documented as a thiamine-responsive/intermittent patient under the code name P.G. (30). "The R230G missense mutation will be described elsewhere. ${ }^{\text {II }}$ Not determined. 
deletion of a $3^{\prime}$-terminal region including the authentic 3 '-splice site (consensus score $91.5 \%$ ) led to the activation of a cryptic site (consensus score 75.5\%) downstream which causes a 21-bp truncation of the exon.

Table III summarizes the occurrence of the E2 mutations in the seven MSUD patients studied. The F215C missense mutation was described previously (9), and the R230G missense mutation will be reported elsewhere. ${ }^{2}$ The remaining five alleles are those documented in this study. The IVS4del[-3.2 kb: -15] allele was originally detected in a compound-heterozygous type II MSUD patient, WG-34, who lived in Montreal, Canada (9). This allele also occurred in a homozygous patient (S.G.) from the same city and in two compound heterozygotes, one in Minnesota (S.B.) and the other in Boston (MGF-497) (30) as described in this study. The relative geographical proximity among these patients suggests a Founder effect for the mutation. Moreover, these patients have either the intermittent/thiamine-responsive (WG-34 and MGF-497) or the classic (S.G. and S.B.) MSUD clinical phenotype, depending on the nature of mutation in the second allele. Of interest is that another classic type II MSUD patient (E.C.), who is compoundheterozygous for the IVS5del[-1:+1] allele and the 90delAT allele, is also responsive to thiamine. We have recently found nine additional E2 alleles, including F215C in WG-34, which occur in several thiamine-responsive MSUD patients. ${ }^{2}$ The data indicate a correlation between a subset of type II MSUD and the thiamine-responsive clinical phenotype. Type II MSUD patients carrying other mutant E2 alleles exhibit the classic (e.g., S.G., S.B., W.J., and A.E.) phenotype (Table III). However, these patients have not been quantitatively studied for thiamine responsiveness. It is probable that additional type II patients with low or unmeasurable BCKD complex activity may still benefit from high doses of thiamine administration with improved tolerance of dietary branched-chain amino acids.

It should also be emphasized that no loci other than E2 of the BCKD complex are affected in documented thiamineresponsive patients that we $(1,9,20)$ or others $(28)$ have studied. The findings strongly suggest that a normal E1 component is a prerequisite for the thiamine-responsive phenotype. The presence of residual BCKD activity in the two type II MSUD patients (WG-34 and MGF-497) is associated with the expression of the mutant E2 protein as detected by Western blotting (Table III). The residual enzyme activity may explain the milder intermittent phenotype in addition to the thiamine responsiveness in these patients. Our working hypothesis is that a normal E2 component, when complexed with E1, is essential for high affinity of the latter enzyme for thiamine pyrophosphate. This may explain the dependence of the responsive MSUD patients on high doses of thiamine $(1,9,20)$. Others have proposed that thiamine pyrophosphate stabilizes enzyme components of the BCKD complex, thereby improving the patient's tolerance for BCAAs (31). The biochemical mechanism for thiamine response in type II patients is currently under investigation.

\section{Acknowledgments}

We thank the following physicians and geneticist for supplying blood samples or cell lines from the type II MSUD patients reported in this

2. Chuang, J.L., R.P. Cox, and D.T. Chuang, manuscript in preparation. study: Dr. Juan Sotos, Dr. Selma Snyderman, Dr. Susan Berry, Dr. Vivian Shih, Dr. Louis Dallaire, and Dr. Grant Mitchell.

This work was supported by grant DK-26758 from the National Institutes of Health.

\section{References}

1. Chuang, D.T., and V.E. Shih. 1995. Disorders of branched-chain amino acid and keto acid metabolism. In The Metabolic and Molecular Bases of Inherited Disease, 7th edition. C.R. Scriver, A.L. Beaudet, W.S. Sly, and D. Valle, editors. McGraw-Hill Inc., New York. 1333-1377.

2. Scriver, C.R., S. MacKenzie, C.L. Clow, and E. Deluin. 1971. Thiamine responsive maple syrup urine disease. Lancet. 1:310-311.

3. Taylor, J., B.H. Robinson, and W.G. Sherwood. 1978. A defect in branched-chain amino acid metabolism in a patient with congenital lactic acidosis due to dihydrolipoyl dehydrogenase deficiency. Pediatr. Res. 12:60-62.

4. Pettit, F.H., S.J. Yeaman, and L.J. Reed. 1978. Purification and characterization of branched-chain $\alpha$-keto acid dehydrogenase complex of bovine kidney. Proc. Natl. Acad. Sci. USA. 75:4881-4885.

5. Yeaman, S.J. 1989. The 2-oxo acid dehydrogenase complexes: recent advances. Biochem. J. 247:625-632.

6. Harris, R.A., R. Paxton, S.M. Powell, G.W. Goodwin, M.J. Kuntz, and A.C. Han. 1986. Regulation of branched-chain $\alpha$-keto acid dehydrogenase complex by covalent modification. Adv. Enzyme Regul. 25:219-237.

7. Zneimer, S.M., K.S. Lau, R.L. Eddy, T.B. Shows, D.T. Chuang, and R.P. Cox. 1991. Regional assignment of two genes of the human branched-chain $\alpha$-keto acid dehydrogenase complex. The E1 $\beta$ gene (BCKDHB) to chromosome $6 \mathrm{p} 21-$ 22 and the E2 gene (DBT) to chromosome 1p31. Genomics. 10:740-747.

8. Lau, K.S., W.J. Herring, J.L. Chuang, M. McKean, D.J. Danner, R.P Cox, and D.T. Chuang. 1992. Structure of the gene encoding dihydrolipoyl transacylase (E2) component of human branched-chain $\alpha$-keto acid dehydrogenase and characterization of an E2 pseudogene. J. Biol. Chem. 267:2409024096.

9. Fisher, C.W., K.S. Lau, C.R. Fisher, R.M. Wynn, R.P. Cox, and D.T. Chuang. 1991. A 17-bp insertion and a Phe215 to Cys missense mutations in the dihydrolipoyl transacylase (E2) mRNA from a thiamine-responsive maple syrup urine disease patient WG-34. Biochem. Biophys. Res. Commun. 174:804809

10. Fisher, C.W., C.R. Fisher, J.L. Chuang, K.S. Lau, D.T. Chuang, and R.P. Cox. 1993. Occurrence of a 2-bp (AT) deletion allele and a nonsense (G-to-T) mutant allele at the E2 (DBT) locus of six patients with maple syrup urine disease: multiple-exon skipping as a secondary effect of the mutations. Am. J. Hum. Genet. 52:414-424.

11. Herring, W.J., M. McKean, N. Dracopoli, and D.J. Danner. 1991. Branched chain acyltransferase absence due to an alu-based genomic deletion allele and an exon skipping allele in a compound heterozygote proband expressing maple syrup urine disease. Biochim. Biophys. Acta. 1138:236-242.

12. Mitsubuchi, H., Y. Nobukuni, I. Akaboshi, Y. Indo, F. Endo, and I. Matsuda. 1991. Maple syrup urine disease caused by a partial deletion in the inner E2 core domain of the branched-chain $\alpha$-keto acid dehydrogenase complex due to aberrant splicing. A single base deletion at a $5^{\prime}$-splice donor site of an intron of the E2 gene disrupts the consensus sequence in this region. J. Clin. Invest. 87:1207-1211.

13. Chuang, D.T., and R.P. Cox. 1988. Enzyme assays with mutant cell lines of maple syrup urine disease. Methods Enzymol. 166:135-146.

14. Miller, G., and M. Lipman. 1973. Release of infectious Epstein-Barr virus by transformed marmoset leukocytes. Proc. Natl. Acad. Sci. USA. 70:190194.

15. Chen, E.Y., and P.H. Seeburg. 1985. Supercoiled sequencing: a fast and simple method for sequencing plasmid DNA. DNA (N.Y.). 4:165-170.

16. Fisher, C.W., J.L. Chuang, T.A. Griffin, K.S. Lau, R.P. Cox, and D.T. Chuang. 1989. Molecular phenotypes in cultured maple syrup urine disease cells. E1 $\alpha$ cDNA sequence and mRNA and subunit contents of the human branched-chain $\alpha$-keto acid dehydrogenase complex. J. Biol. Chem. 264:34483453 .

17. Chuang, J.L., J.R. Davie, J.M. Chinsky, R.M. Wynn, R.P. Cox, and D.T Chuang. 1995. Molecular and biochemical basis of intermediate maple syrup urine disease. Occurrence of homozygous G245R and F364C mutations at the E1 $\alpha$ locus of Hispanic-Mexican patients. J. Clin. Invest. 95:954-963.

18. Krawczak, M., J. Reiss, and D.N. Cooper. 1992. The mutational spectrum of single base pair substitutions in mRNA splice junctions of human genes: causes and consequences. Hum. Genet. 90:41-54.

19. Nakai, K., and H. Sakamoto. 1994. Construction of a novel database containing aberrant splicing mutations of mammalian genes. Gene (Amst.). 141: $171-177$.

20. Cox, R.P., J.L. Chuang, and D.T. Chuang. 1997. Maple syrup urine disease. Clinical and molecular genetic considerations. In The Molecular and Genetic Basis of Neurological Disease. 2nd edition. R.N. Rosenberg, S.B. Prusiner, S. DiMauro, and R.L. Barchi, editors. Butterworth-Heinemann, Boston. 1175-1193. 
21. Krawczak, M., and D. Cooper. 1991. Gene deletions causing human genetic disease: mechanisms of mutagenesis and the role of the local DNA sequence environment. Hum. Genet. 86:425-441.

22. Chuang, J.L., C. R. Fisher, R.P. Cox, and D.T. Chuang. 1994. Molecular basis of maple syrup urine disease: novel mutations at the E1 $\alpha$ locus that impairs E1 $\left(\alpha_{2} \beta_{2}\right)$ assembly or decreases steady-state E1 $\alpha$ mRNA levels of branched-chain $\alpha$-keto acid dehydrogenase complex. Am J. Hum. Genet. 55: 297-304.

23. Berget, S.M. 1995. Exon recognition in vertebrate splicing. J. Biol. Chem. 270:2411-2414.

24. Shapiro, M.B., and P. Senapathy. 1987. RNA splice junctions of different classes of eukaryotes: sequence statistics and functional implications in gene expression. Nucleic Acids Res. 15:7155-7174.

25. Maquat, L. 1996. Defects in RNA splicing and the consequence of shortened translational reading frames. Am. J. Hum. Genet. 59:279-286.

26. Nichols, R.C., O. Rudolphi, B. Ek, R. Exelbert, P.H. Plotz, and N. Raben. 1996. Glycogenosis type VII (Tarui disease) in a Swedish family: two novel mutations in muscle phosphofructokinase gene (PFK-M) resulting in intron re- tentions. Am. J. Hum. Genet. 59:59-65.

27. Lau, K.S., J.L. Chuang, W.J. Herrring, D.J. Danner, and D.T. Chuang 1992. The complete cDNA sequence for dihydrolipoyl transacylase (E2) of human branched-chain $\alpha$-keto acid dehydrogenase. Biochim. Biophys. Acta 1132: 319-321.

28. Ellerine, N.P., W.J. Herring, L.J. Elsas II, M.C. McKean, P.D. Klein, and D.J. Danner. 1993. Thiamin-responsive maple syrup urine disease in a patient antigenically missing dihydrolipoamide acyltransferase. Biochem. Med. Metab. Biol. 49:363-374.

29. Ad Hoc Committee on Mutation Nomenclature. 1996. Update on nomenclature for human mutation. Hum. Mutat. 8:197-202.

30. Pueschel, S.M., M.J. Bresnan, V.E. Shih, and H.L. Levy. 1979. Thiamine-responsive intermittent branched-chain ketoaciduria. J. Pediatr. 94:628631.

31. Danner, D.J., S.K. Lemon, and L.J. Elsas, II. 1980. Stabilization of mammalian liver branched-chain $\alpha$-ketoacid dehydrogenase by thiamin pyrophosphate. Arch. Biochem. Biophys. 202:23-28. 